(C) [2009] IEEE. Reprinted, with permission, from [Wei Xu, Jianguo Zhu, Yongchang Zhang, D. G Dorrell, Yaohua Li and Yongjian Li, An Improved Series Equivalent Circuit of a Single-Sided Linear Induction Motor, Industrial Electronics, 2009. IECON '09. 35th Annual Conference of IEEE, 3-5 Nov. 2009]. This material is posted here with permission of the IEEE. Such ermission of the IEEE does not in any way imply IEEE endorsement of any of the University of Technology, Sydney's products or services. Internal or personal use of this material is permitted.

However, permission to reprint/republish this material for advertising or promotional purposes or for creating new collective works for resale or redistribution must be obtained from the IEEE by writing to pubs-permissions@ieee.org. By choosing to view this document, you agree to all provisions of the copyright laws protecting it 


\title{
An Improved Series Equivalent Circuit of a Single-Sided Linear Induction Motor
}

\author{
Wei Xu ${ }^{1}$, Jianguo Zhu ${ }^{1}$, Yongchang Zhang ${ }^{1}$, D. G Dorrell ${ }^{1}$, Yaohua $\mathrm{Li}^{2}$, Yongjian $\mathrm{Li}^{1}$ \\ ${ }^{1}$ School of Electrical, Mechanical and Mechatronic Systems, University of Technology,Sydney, Australia \\ ${ }^{2}$ Institute of Electrical Engineering, Chinese Academy of Sciences, Beijing P. R. China \\ wxu@eng.uts.edu.au
}

\begin{abstract}
The derivation of the equivalent circuit for a singlesided linear induction motor (SLIM) is not straightforward. Especially if it includes longitudinal end effects from the cutopen primary magnetic path, transverse edge effects from the differing widths between the primary lamination and secondary sheet, and half filled primary slots. This paper proposes an improved series equivalent circuit for this machine. The longitudinal end effects are estimated using three different impedances representing the normal, forwards and backwards flux density waves in the air-gap. The transverse edge effects accounted for with a correction coefficient $K_{\mathrm{t}}$ and an air-gap flux density correction coefficient $K_{\mathrm{b}}$. Using the series circuit, the performance of the SLIM was assessed in a similar manner to a rotating induction machine. A $4 \mathrm{~kW}$ SLIM prototype was tested which validated the simulation technique.
\end{abstract}

\section{INTRODUCTION}

Single-sided linear induction motors (SLIMs) are derived from rotating induction motors (RIMs). They can be utilized in a number of industrial systems; they have obvious application in rapid light transportation and even in very low speed low-head hydro (water-wheel) generation (where the copper or aluminum secondary plate is on the large-diameter wheel). In transportation systems, they have the advantage (over an RIM) of the propulsion thrust being achieved without reliance on friction between wheel and rail (allowing high inclines) as well as several others [1, 2]. While most current literature for new transport or renewable energy applications consider various forms of permanent-magnet machine for the electromechanical energy conversion unit, the linear machine may have niche applications in these areas.

In a rotating machine, an accurate equivalent circuit model can be obtained with reasonable ease because of pole symmetry. The model of the linear equivalent is more complicated because some parameters in the circuit are asymmetrical [3], i.e., it has transverse and longitudinal end effects. The transverse edge effects result in changes in the transverse air-gap flux density. The longitudinal end effects are derived from the primary discontinuous magnetic circuit where the electrical conditions can vary greatly. When a double layer winding is used some of the end slots will only contain one coil side so that they with be only half filled.

There have been many studies conducted on the SLIM; many make approximations. For example, exit end-wave analysis is not considered in [3], half-filled slots are not taken into account in [4], and transverse end effects are ignored in [5]. Such assumptions generate errors in the analysis of the SLIM performance. This paper uses the air-gap flux density

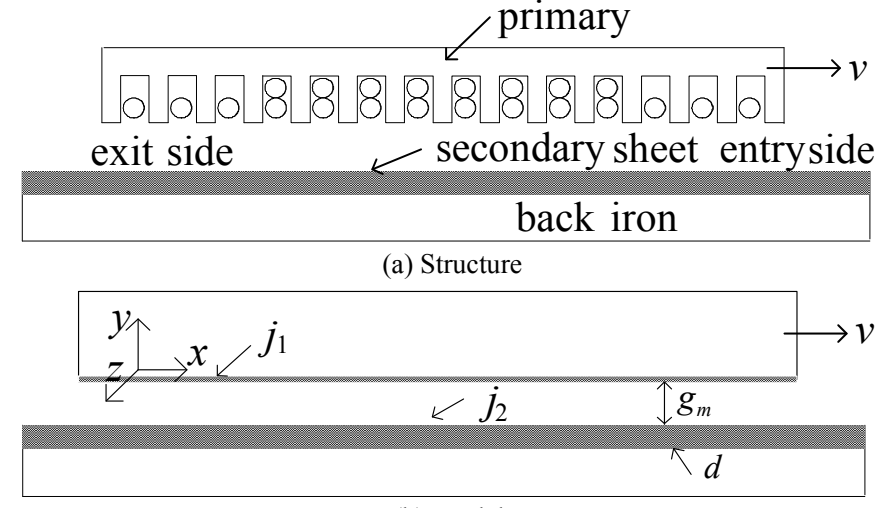

(b) Model

Fig. 1. The structure and one-dimensional analytic model of SLIM. (a).Structure.(b).Model.

equation develop an improved series equivalent circuit which considers the influences of the entry and exit end-wave flux waves in the air-gap, as well as transverse effects, half filled slots and air-gap leakage flux.

\section{THE PHYSICAL MODEL}

To simplify the analysis, some assumptions are used [6-12]: (a) the stator iron has infinite permeability;

(b) skin effect is neglected in the secondary;

(c) winding space harmonics are negligible; and

(d) the primary and secondary currents flow in infinitesimally thin sheets.

The structure and one-dimensional representation of the SLIM are shown in Fig. 1. The model described in [11] derives several relevant coefficients for the secondary sheet current and air-gap flux density. The analysis from [12] can be used to analyze the system. From Ampere's Law (with reference to the geometry in Fig. 1):

$$
\frac{g}{\mu_{0}} \frac{\partial b_{y}}{\partial x}=j_{1}+j_{2}
$$

We can implement a one-dimensional Curl function for the secondary plate - the air-gap electric field has to be referred to the secondary:

$$
\frac{\partial e_{z}^{\prime}}{\partial x}=\frac{\partial b_{y}}{\partial t}+\frac{\partial b_{y}}{\partial x} \frac{\partial x}{\partial t}=\frac{\partial b_{y}}{\partial t}+v \frac{\partial b_{y}}{\partial x}
$$

where the second term on the right hand side is the speed term. What we now want to try and do is refer the secondary current sheet to the primary so that we can obtain an expression for the air-gap flux density in terms of the (measureable) primary current. The secondary consists of a 
copper conducting sheet and we will assume that the current is resistance-limited so that for a plate of thickness $d$ and surface conductivity $\sigma$ :

$$
e_{z}^{\prime}=\frac{j_{2}}{\sigma}
$$

where $\sigma=d / \rho$ (in ohm) and $\rho$ is the volume resistivity (in ohm-m). We can combine these equations so that

$$
g \frac{\partial^{2} b_{y}}{\partial x^{2}}-\sigma \mu_{0} v \frac{\partial b_{y}}{\partial x}-\sigma \mu_{0} \frac{\partial b_{y}}{\partial t}=\mu_{0} \frac{\partial j_{1}}{\partial x}
$$

If we assume that

$$
j_{1}=j_{1}(x, t)=\operatorname{Re}\left\{\bar{J}_{1} e^{j(\omega t-\pi x / \tau)}\right\}
$$

then we can obtain an expression for the air-gap flux density:

$$
g \frac{\partial^{2} b_{y}}{\partial x^{2}}-\sigma \mu_{0} v \frac{\partial b_{y}}{\partial x}-j \sigma \mu_{0} \omega b_{y}=\operatorname{Re}\left\{-j \mu_{0} \frac{\pi}{\tau} J_{1} e^{j(\omega t-\pi x / \tau)}\right\}
$$

The solution of $b_{\mathrm{y}}$ is

$$
\begin{aligned}
b_{y} & =b_{y}(x, t) \\
& =\operatorname{Re}\left\{\left(\bar{B}_{0} e^{-j \frac{\pi x}{\tau}}+\bar{B}_{1} e^{-\frac{x}{\alpha_{1}}} e^{-j \frac{\pi x}{\tau_{e}}}+\bar{B}_{2} e^{\frac{x}{\alpha_{2}}} e^{j \frac{\pi x}{\tau_{e}}}\right) e^{j \omega t}\right\}
\end{aligned}
$$

We can derive the air-gap flux density coefficients so that

$$
\bar{B}_{0}=\frac{j \tau \mu_{0} \bar{J}_{1}}{g \pi(1+j s G)}
$$

where $G=\frac{\sigma \mu_{0} \omega \tau^{2}}{g \pi^{2}}$

and the $\operatorname{slip} s=\left(v_{s}-v\right) / v_{s}$; we need to define the synchronous velocity $v_{s}=\omega \tau / \pi$ with pole pitch $\tau$. The second term is

$$
\bar{B}_{1}=j \frac{\tau}{\pi}\left(\frac{1}{\alpha_{1}}+j \frac{\pi}{\tau_{e}}\right) \bar{B}_{0}
$$

where $\alpha_{1}=\frac{2 g}{X g-\sigma \mu_{0} v}$ and $\tau_{e}=\frac{2 \pi}{Y}$.

We can define $X+j Y=\sqrt{\left(\frac{\sigma \mu_{0} v}{g}\right)^{2}+4 j \frac{\sigma \mu_{0} \omega}{g}}$.

In a similar fashion

$$
\bar{B}_{2}=-j \frac{\tau}{\pi} e^{-\left(\frac{1}{\alpha_{2}}+j \frac{\pi}{\tau_{e}}\right) L_{p}}\left(\frac{1}{\alpha_{2}}+j \frac{\pi}{\tau_{e}}\right) \bar{B}_{0}
$$

where $\alpha_{2}=\frac{2 g}{X g+\sigma \mu_{0} v}$.

We now have a complete definition of the air-gap fluxdensity wave in terms of the stator surface current density. Most parameter definitions from (1) to (10) are given in the appendix. In (2), the flux density $b_{\mathrm{y}}$ includes three parts: $b_{0}$, $b_{1}$ and $b_{2} . b_{0}$ is the normal traveling wave which moves forward in a similar manner to the fundamental flux density wave in a rotating induction machine (RIM). $b_{1}$ and $b_{2}$ are determined from the boundary conditions, which are the entrance and exit end-effect waves [9-11]. $b_{1}$ is a gradually

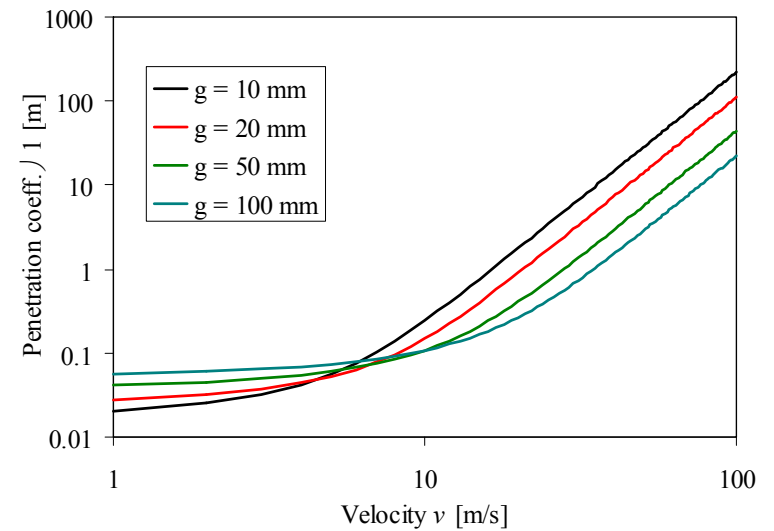

(a)

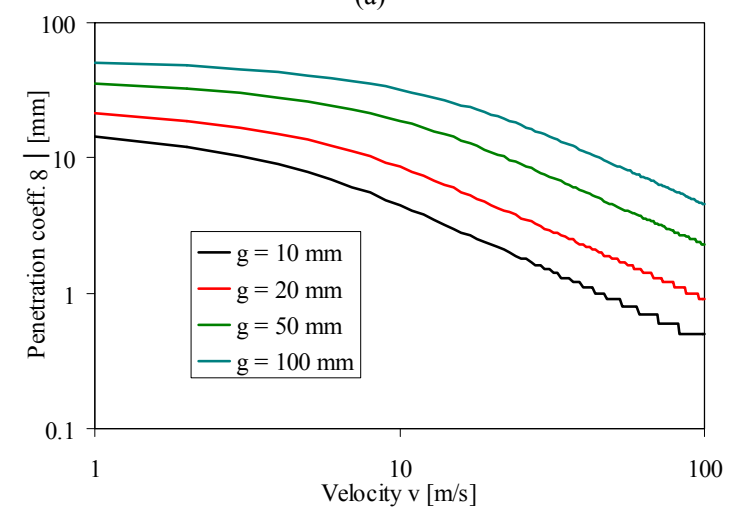

(b)

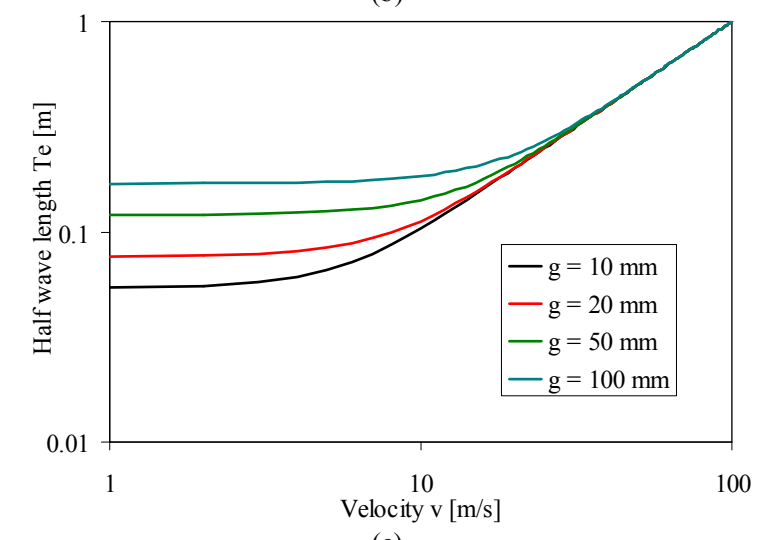

(c)

Fig. 2. Penetration coefficients and half-wave length versus motor velocity. (a) Entry penetration coefficient $\alpha_{1}$. (b) Exit penetration coefficient $\alpha_{2}$. (c) Half-wave length $\tau_{\mathrm{e}}$.

attenuating wave traveling along the $x$-axis. Its attenuation constant is $1 / \alpha_{1}$ and end-effect half-wave length is $\tau_{\mathrm{e}}$. $b_{2}$ travels along the $x$-axis in the negative direction with an attenuation constant of $1 / \alpha_{2}[10]$.

The curves for $\alpha_{1}, \alpha_{2}$ and $\tau_{\mathrm{e}}$ are shown in Fig. 2. It is assumed that the SLIM operates from zero to $100 \mathrm{~m} / \mathrm{s}$, and the secondary copper sheet equivalent surface resistivity $(=\rho / d)$ is $5.63 \times 10^{-5} \mathrm{ohm}$ where the plate thickness $d$ is $3 \mathrm{~mm}$. From Fig. 2 (a), the length of the entry wave penetration coefficient $\alpha_{1}$ increases with motor velocity $v$. It is close to $0.4 \mathrm{~m}$ when equivalent air-gap length $g$ is $10 \mathrm{~mm}$ and $v$ is 10 $\mathrm{m} / \mathrm{s}$. However, the length of the entry wave penetration coefficient $\alpha_{2}$ in Fig. 2 (b) decreases with increasing motor velocity, and it is almost $2 \mathrm{~mm}$ when $g$ is $10 \mathrm{~mm}$ and $v$ is 10 


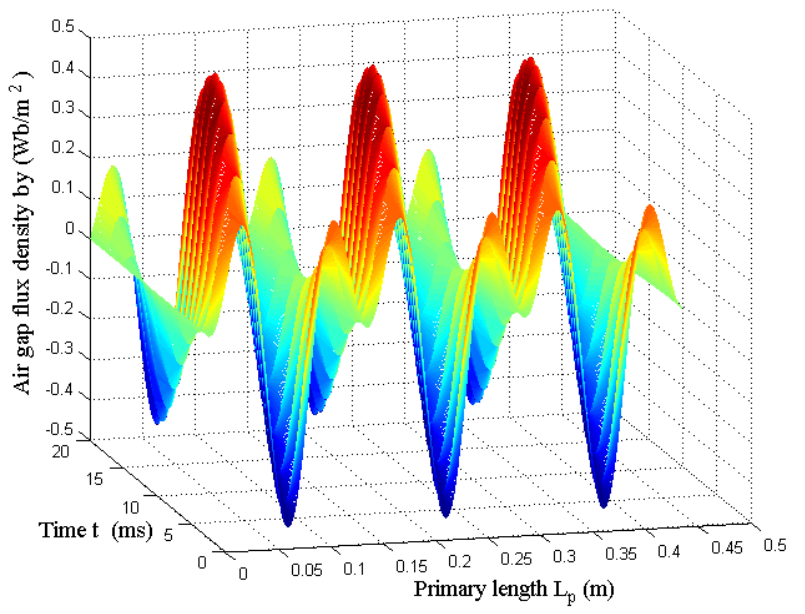

Fig. 3. Air gap flux density distribution in the primary length (6-pole wave progression illustrated).

$\mathrm{m} / \mathrm{s}$. From Fig. 2 (c), the half-wave length $\tau_{\mathrm{e}}$ of the end-effect wave increases with velocity, is close to $0.1 \mathrm{~m}$ when $g$ is 10 $\mathrm{mm}$ and $v$ is $10 \mathrm{~m} / \mathrm{s}$.

Fig. 3 shows a SLIM air-gap flux density distribution for the machine studied here over the primary length area. The final flux wave is not sinusoidal because of the influence of the entry and exit flux waves.

The electric field intensity in the air-gap is denoted by (2). However this now needs to be referred to primary so that the speed term is ignored. Hence

$e_{z}(x, t)=\int \frac{\partial b_{y}}{\partial t} \partial x=\operatorname{Re}\left\{\begin{array}{l}\bar{E}_{0} e^{j\left(\omega t-\frac{\pi x}{\tau}\right)}+\bar{E}_{1} e^{-\frac{x}{\alpha_{1}}} e^{j\left(\omega t-\frac{\pi x}{\tau_{e}}\right)} \\ +\bar{E}_{2} e^{\frac{x}{\alpha_{2}}} e^{j\left(\omega t+\frac{\pi x}{\tau_{e}}\right)}\end{array}\right\}$

With knowledge of the air-gap flux density distribution, the electric field intensity produces three terms: $\boldsymbol{E}_{0}, \boldsymbol{E}_{1}$ and $\boldsymbol{E}_{2}$. These individually produce an induced electromotive force in the machine windings so that

$$
\begin{aligned}
& \bar{E}_{0}=-\omega \frac{\tau}{\pi} \bar{B}_{0}=-v_{s} \bar{B}_{0} \text { and } \\
& \bar{E}_{1}=-\frac{j \omega \bar{B}_{1} \alpha_{1} \tau_{e}}{\left(\tau_{e}+j \pi \alpha_{1}\right)}, \bar{E}_{2}=\frac{j \omega \bar{B}_{2} \alpha_{2} \tau_{e}}{\tau_{e}+j \pi \alpha_{2}} .
\end{aligned}
$$

\section{EQUIVALENT CIRCUIT}

The equivalent circuit is essential for analyzing the motor and predicting its performance. It is particularly necessary for the initial SLIM electromagnetic design procedure. Here the induced electromotive force is used to compute the fundamental-wave impedance. The complex power and electric-magnetic thrust methods are adapted to derive the entrance- and exit-wave impedances, which are utilized to calculate the SLIM thrust, power factor, efficiency and other performance parameters.

\section{A. Fundamental-wave Impedance}

The fundamental elements such as excitation current, electric field intensity and flux density are assumed to be sinusoidal shown below. From [11], the entrance and exitwave elements, such as $b_{1}(x, t), b_{2}(x, t), e_{1}(x, t), e_{2}(x, t)$, have similar forms. We can use the following equations to represent the current, air-gap flux density and electric fields:

$$
\begin{aligned}
& j(x, t)=\operatorname{Re}\left\{\bar{J}_{1} e^{j\left(\omega t-\frac{\pi x}{\tau}\right)}\right\} \\
& b_{0}(x, t)=\operatorname{Re}\left\{\bar{B}_{0} e^{j\left(\omega t-\frac{\pi x}{\tau}\right)}\right\} \\
& e_{0}(x, t)=\operatorname{Re}\left\{\bar{E}_{0} e^{j\left(\omega t-\frac{\pi x}{\tau}\right)}\right\}
\end{aligned}
$$

The magnetic motive force $F(x, t)$ is of a similar form:

$$
F(x, t)=\operatorname{Re}\left\{j \frac{g \tau}{\pi} J_{1} e^{j\left(\omega t-\frac{\pi x}{\tau}\right)}\right\}
$$

The relationship between primary current sheet $J_{1}$ and phase current $I$ is

$$
\bar{J}_{1}=j J_{1}=\frac{\sqrt{2} m w_{1} K_{w 1}}{\tau P} I
$$

where we assume that the reference current is real. The induced phase electromotive force is

$$
\bar{\varepsilon}_{0}=j \sqrt{2} w_{1} K_{w 1} l_{w} \bar{E}_{0}
$$

The fundamental-wave impedance can be calculated:

$$
\bar{Z}_{m}=\frac{\bar{\varepsilon}_{0}}{I}=\frac{j X_{m}}{(1+j s G)}
$$

where $X_{\mathrm{m}}$ is

$$
X_{m}=\frac{4 m f\left(w_{1} K_{w_{1}}\right)^{2} l_{w} \tau \mu_{0}}{P \pi g}
$$

The fundamental-wave thrust $F_{\mathrm{e} 0}$ is denoted by

$F_{e 0}=\frac{l_{w}}{2} \operatorname{Re} \int_{0}^{L_{p}} \bar{B}_{0} e^{-j \frac{\pi x}{\tau}}\left\{\bar{J}_{1} e^{-j \frac{\pi x}{\tau}}\right\}^{*} . d x=\frac{m I^{2} \operatorname{Re}\left\{\bar{Z}_{m}\right\}}{v_{s}}$

where the integral length along the primary length $L_{p}=2 \tau P$. The mechanical power can be obtained from (20) by multiplying by the speed $v_{r}$. But $v_{r}=(1-\mathrm{s}) v_{s}$ so that the mechanical power becomes

$$
P_{m 0}=m I^{2} \operatorname{Re}\left\{(1-s) \bar{Z}_{m}\right\}
$$

We can investigate $\boldsymbol{Z}_{m}$ in (18). If we follow through some rearrangement of (18) then we obtain

$$
\bar{Z}_{m}=\frac{j X_{m}}{(1+j s G)}=\frac{j X_{m}\left(r_{2} / s\right)}{\left(r_{2} / s+j X_{m}\right)}=\frac{j X_{m}}{\left(1+s j X_{m} / r_{2}\right)}
$$

which can be represented by the equivalent circuit in Fig. 4(b). From this the rotor resistance (referred to the stator) is

$$
r_{2}=\frac{2 m\left(w_{1} K_{w 1}\right)^{2} l_{w}}{\sigma \tau P}
$$


This derivation illustrates that the theory is similar to the standard equivalent circuit analysis for rotating machines. Because the secondary is a simple cooper plate then the secondary inductance is assumed negligible and the current is resistance limited.

\section{B. Entrance-wave Impedance}

The electromagnetic power $P_{m 1}$ for the $y$-axis entrance flux wave is the power flowing into the rotor through this wave:

$$
P_{m 1}=\frac{l_{w}}{2} \operatorname{Re} \int_{0}^{L_{p}}-\bar{E}_{1} e^{-\frac{x}{\alpha_{1}}} e^{-j \frac{\pi x}{\tau_{e}}} \cdot\left\{\bar{J}_{1} e^{-j \frac{\pi x}{\tau}}\right\}^{*} \cdot d x
$$

so that

$$
P_{m 1}=\operatorname{Re}\left\{m I^{2}\left(K_{1} \bar{Z}_{m}\right)\right\}
$$

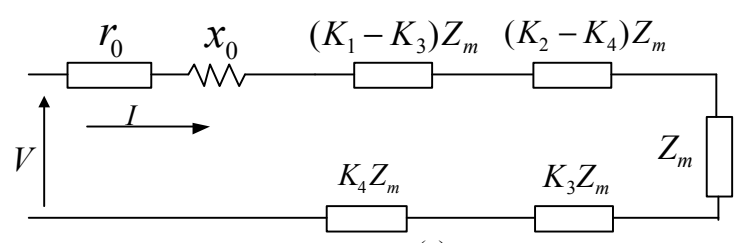

(a)

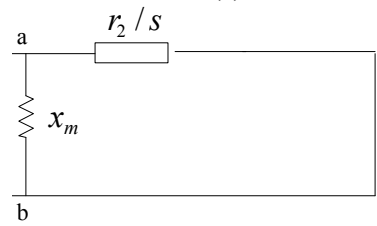

(b)

Fig. 4. The series equivalent circuit.(a)Per-phase circuit.(b) $\boldsymbol{Z}_{m}$ representation

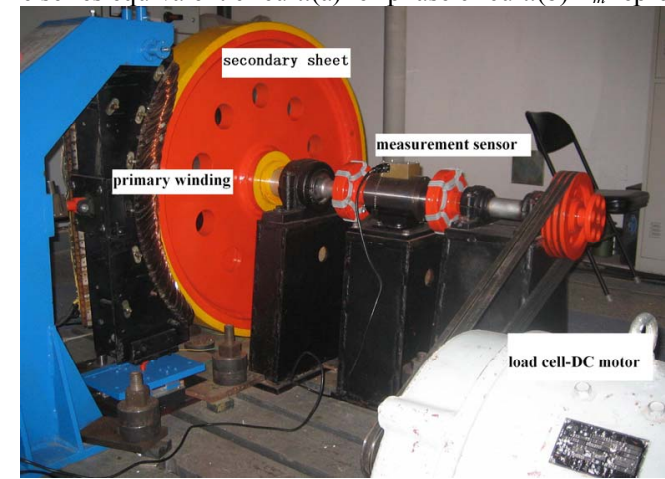

Fig. 5. The SLIM prototype.

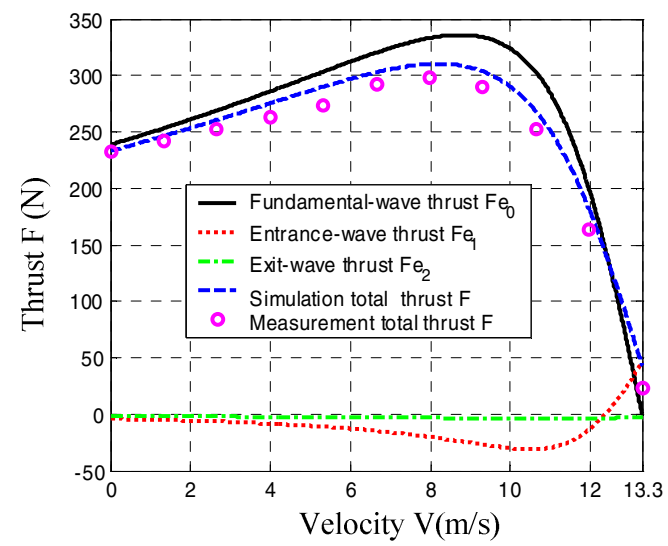

Fig. 6. The thrusts resulted from different air gap flux densities. where

$$
K_{1}=-\frac{\alpha_{1}}{2 P}\left(\frac{\tau_{e}}{\tau \tau_{e}+j \alpha_{1} \pi\left(\tau-\tau_{e}\right)}\right) .
$$

However, the entrance-wave thrust $F_{\mathrm{el}}$ can be used to derive how much of $P_{m 1}$ is converted into mechanical power. This is similar to (20):

$$
F_{e 1}=\frac{l_{w}}{2} \operatorname{Re} \int_{0}^{L_{p}} \bar{B}_{1} e^{-\frac{x}{\alpha_{1}}} e^{-j \frac{\pi x}{\tau_{e}}}\left\{\bar{J}_{1} e^{-j \frac{\pi x}{\tau}}\right\}^{*} \cdot d x=\frac{m I^{2} \operatorname{Re}\left\{K_{3} \bar{Z}_{m}\right\}}{v_{s}}
$$

which becomes

$$
P_{e 1}=m I^{2} \operatorname{Re}\left\{(1-s) K_{3} \bar{Z}_{m}\right\}
$$

where

$$
K_{3}=\frac{-1}{2 P} \cdot \frac{1}{\tau \tau_{e}+j \alpha_{1} \pi\left(\tau-\tau_{e}\right)} \cdot \frac{\tau\left(\tau_{e}+j \alpha_{1} \pi\right)}{j \pi} .
$$

The total input impedance of the entrance wave is $K_{1} \boldsymbol{Z}_{\mathrm{m}}$. For (26), the thrust (main) impedance is $K_{3} Z_{\mathrm{m}}$. Therefore the impedance (which does not contribute to the thrust) can be defined as $\left(K_{1}-K_{3}\right) Z_{\mathrm{m}}$, this is called the entrance-wave leakage impedance. These are illustrated in the series equivalent circuit in Fig. 4(a).

\section{Exit-wave Impedance}

The exit-wave electromagnetic power $P_{m 2}$ for the exit-end flux density wave can be obtained in a similar manner:

$$
P_{m 2}=m I^{2}\left(K_{2} \bar{Z}_{m}\right)
$$

where the constant is

$$
K_{2}=-\frac{\alpha_{2}}{2 P} \frac{\tau_{e}}{\tau \tau_{e}+j \alpha_{2} \pi\left(\tau+\tau_{e}\right)} .
$$

The exit-wave thrust $F_{e 2}$ is

$$
F_{e 2}=\frac{m I^{2} \operatorname{Re}\left\{K_{4} \bar{Z}_{m}\right\}}{v_{s}}
$$

where

$$
K_{4}=-j \frac{\alpha_{1}}{2 P} \frac{\tau\left(\tau_{e}+j \alpha_{2} \pi\right)}{\alpha_{2} \pi\left[\tau \tau_{e}+j \alpha_{2} \pi\left(\tau+\tau_{e}\right)\right]} .
$$

Again, we can divide the impedances into two. The exitwave equivalent impedance is $K_{2} \boldsymbol{Z}_{\mathrm{m}}$ but the exit-wave main impedance is $K_{4} \boldsymbol{Z}_{\mathrm{m}}$, while the exit-wave leakage impedance is $\left(K_{2}-K_{4}\right) Z_{\mathrm{m}}$. These are illustrated in Fig. 4(a).

\section{Equivalent Circuit and Calculation}

The equivalent circuit is shown in Fig. 4. From (24), (27), (29) and (31) it can be shown (after considerable manipulation) that

$$
\begin{aligned}
K_{1}+K_{2} & =K_{3}+K_{4} \\
& =\frac{-\left[\left(\alpha_{1}+\alpha_{2}\right) \tau_{e}^{2}+j 2 \pi \alpha_{1} \alpha_{2} \tau \tau_{e}\right]}{2 P\left[\tau \tau_{e}+j \alpha_{1} \pi\left(\tau-\tau_{e}\right)\right]\left[\tau \tau_{e}+j \alpha_{2} \pi\left(\tau+\tau_{e}\right)\right]}
\end{aligned}
$$

This illustrates that the sum of entrance and exit leakage impedances is zero because the total secondary input power $\left(K_{1}+K_{2}\right.$ term) is equal to the total mechanical output power $\left(K_{3}+K_{4}\right.$ term). Therefore the total equivalent longitudinal end-effect impedance $Z_{\mathrm{L}}$ is 


$$
\bar{Z}_{L}=K_{L} \bar{Z}_{m}
$$

where

$$
K_{L}=K_{1}+K_{2}
$$

It is necessary to adjust the $z$-axis current in the secondary conducting sheet and $y$-axis flux density in the air-gap. These corrections are called the transverse different-width correction coefficient $K_{\mathrm{t}}$ and the air-gap flux density correction coefficient $K_{\mathrm{b}}$. These are analogous to end-ring and fringing effects in rotating induction motors. The half-filled slots at the ends of the machine are accounted for by use of the half-filled slot correct coefficient $K_{\mathrm{p}}$. These coefficients lead to small corrections in the fundamental and longitudinal end-effect impedances which make the performance analysis more accurate [13].

The modified fundamental impedance $\boldsymbol{Z}_{\mathrm{mc}}$ is

$$
\bar{Z}_{m c}=K_{p} K_{t} K_{b} \bar{Z}_{m}
$$

and modified longitudinal end-effect impedance $\boldsymbol{Z}_{\mathrm{Lc}}$ becomes

$$
\bar{Z}_{L c}=K_{p} K_{L} \bar{Z}_{m} \text {. }
$$

This leads to the total impedance $\boldsymbol{Z}_{\mathrm{t}}$ where

$$
\bar{Z}_{t}=r_{0}+j x_{0}+\bar{Z}_{m c}+\bar{Z}_{L c}
$$

and the total thrust $F$ is modified so that

$$
F=\frac{m I^{2} \operatorname{Re}\left\{\bar{Z}_{m c}+\bar{Z}_{L c}\right\}}{v_{s}} .
$$

The power factor $\cos \varphi$ is important in induction machines and this can be obtained from

$$
\cos \varphi=\cos [\tan (\operatorname{Im}(\bar{Z}) / \operatorname{Re}(\bar{Z}))]
$$

with the efficiency $\eta$ calculated from

$$
\eta=\operatorname{Re}\left\{\bar{Z}_{m c}+\bar{Z}_{L c}\right\} \frac{(1-s)}{\operatorname{Re}\left\{\bar{Z}_{t}\right\}} .
$$

\section{SIMULATION AND EXPERIMENTATION}

In order to validate the series equivalent circuit analysis developed in the previous section, the algorithm was simulated and experimental verification obtained from a SLIM prototype. The test rig is shown in Fig. 5. A rotating copper wheel arrangement was used to represent an infinitely long secondary sheet. The dimensions of the machine are given in Table I. The machine was a $4 \mathrm{~kW} 220 \mathrm{~V} 3$-phase 50 $\mathrm{Hz}$ 6-pole linear induction machine with a synchronous speed of $13.3 \mathrm{~m} / \mathrm{s}$ at $50 \mathrm{~Hz}$. The supply was a variable-frequency inverter source so that a set of torque/speed curves could be obtained for a variety of frequencies.

TABLE I

THE DIMENSIONS OF THE SLIM

\begin{tabular}{|l|c|}
\hline Pole pitch [m] & 0.113 \\
\hline Number of pole pairs & 3 \\
\hline Secondary width [m] & 0.17 \\
\hline Secondary sheet thick [mm] & 3 \\
\hline Frequency [Hz] & 50 \\
\hline Thrust [N] & 330 \\
\hline Primary length/width [m] & $0.45 / 0.1$ \\
\hline Air gap length [mm] & 7 \\
\hline Primary phase voltage [V] & 220 \\
\hline
\end{tabular}

The machine was loaded with a DC machine connected to the SLIM via belts. The DC machine could operate at any desired speed including operation at the synchronous speed (driven by the load in motoring mode). The system could measure the SLIM velocity, load power and thrust. Other variables such as

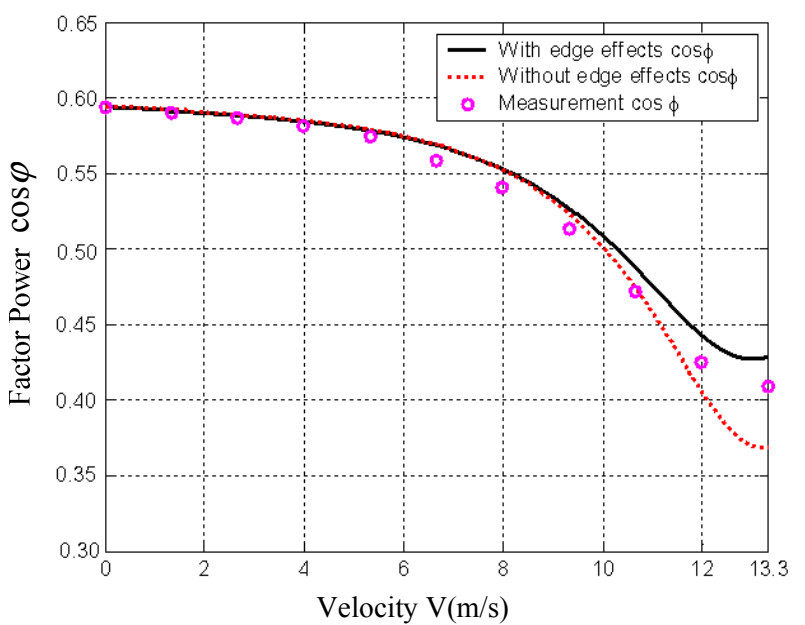

Fig. 7. The power factor curves.

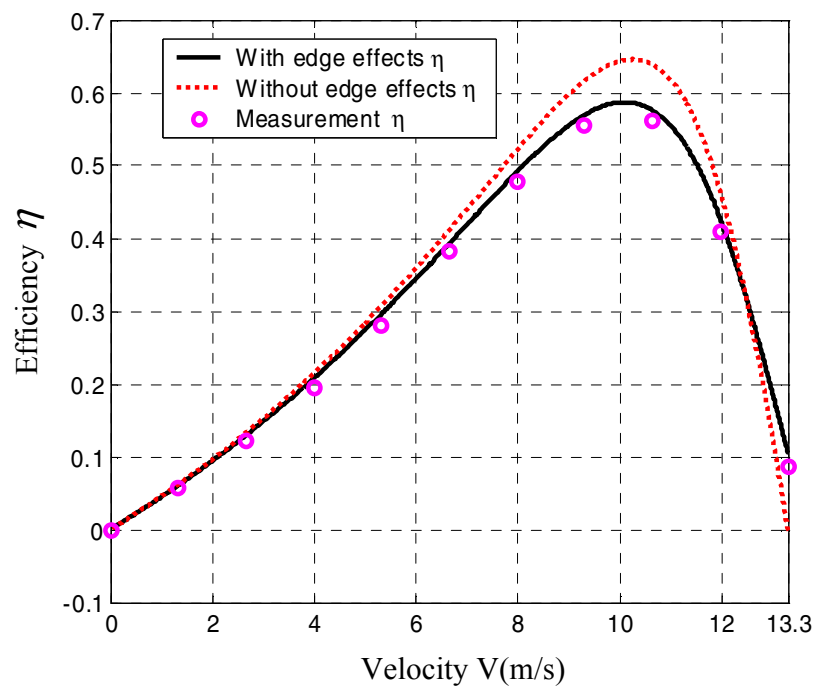

Fig. 8. The efficiency curves.

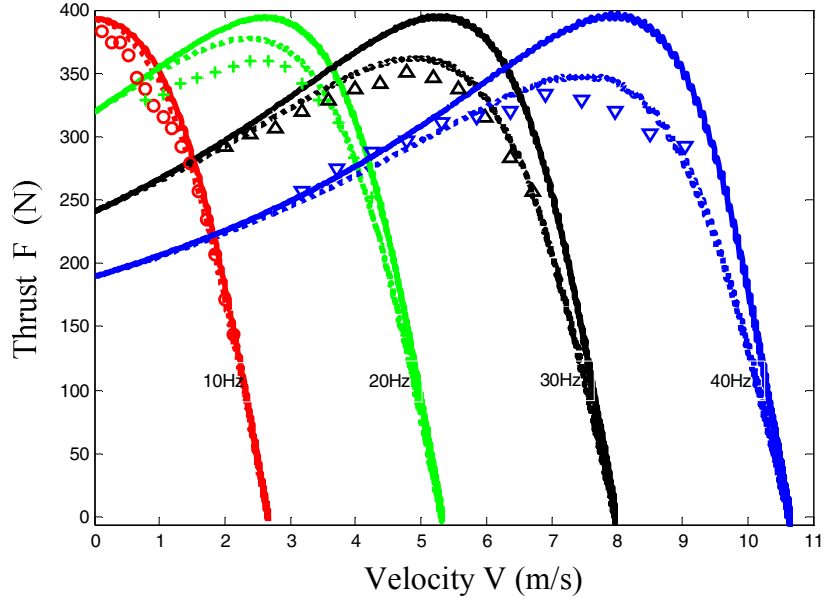

Fig. 9. The thrust curves in constant current and different frequencies. 
the primary phase current and phase voltage were recorded using a power analyzer [10].

The simulation and experimental results for the thrust, power factor, and efficiency are given in Figs. 6, 7 and 8 for a constant input primary voltage of $220 \mathrm{~V}$ and a frequency of $50 \mathrm{~Hz}$. The trust/velocity curves shown in Fig. 6 are very similar to those of a rotating cage induction machine. The entry-wave thrust is negative except over a small slip region. The exit-wave thrust is very small over the whole operational region. The total calculated thrust $F$ curve, which is the sum of the three derived thrust components, agrees well with the measured points. The net thrust curve is lower than the fundamental curve due to the entry-wave thrust (apart from close to synchronism where the entry-thrust becomes positive). This illustrates that consideration of the fundamental flux wave only is insufficient for accurate analysis. In Figs. 7 and 8, the power factor and efficiency are influenced little by the end effects in the lower speed region. As the velocity increases and the entry-wave thrust becomes positive near the synchronous speed, the end effects do begin to affect the power factor and efficiency to a greater extent. These simulations are consistent with the results found in [11]. A peak efficiency of about $60 \%$ was found. While this seems low it is reasonable for a small robust induction machine.

Fig. 9 shows the thrust curves with a constant primary current of $15 \mathrm{~A}$ at varying frequencies from 0 to $40 \mathrm{~Hz}$. The solid lines represent the thrusts without end effects in the simulation, the dashed lines represent the thrusts with end effects in the simulation and the markers represent the measured. The thrusts at low velocity (high slip) are not affected by the end effects. As the speed increases then end effects have an increasing influence on the thrust across all the different constant-frequency curves.

\section{CONCLUSIONS}

This paper uses an equation for the air-gap flux density and develops a convenient series equivalent circuit to calculate the performance, including thrust, efficiency and power factor, for a single-sides induction machine. The model is comprehensive and considers the longitudinal end-effects, transverse edge-effects and half-filled slots. Test data from a $4 \mathrm{~kW}$ SLIM prototype is put forward to validate the simulation results and good correlation was found. This model could also be implemented to simulate high power SLIMs and would be useful for designing linear machines for use in applications such a rapid transport or even low-speed low-head hydro water wheels where it would be used in generating mode.

\section{REFERENCES}

[1] I. E. Davidson and J. F. Gieras, "Performance Analysis of a ShadedPole Linear Induction Motor Using Symmetrical Components, Field Analysis, and Finite Element Method", IEEE Trans. on Energy Conversion, Vol.15, No.1, pp. 24-29, March 2000.

[2] S. Nonaka and T. Higuchi, "Design of single-sided linear induction motors for urban transit," IEEE Transaction on Vehicular Technology, Vol.37, No.3, August 1988.

[3] A. K. Rathore and S. N. Mahendra, "Simulation of secondary flux oriented control of linear induction motor considering attraction force and transverse edge effect," International Conference on Electrical Engineering Proceeding, pp.158-163, July 2003.

[4] J. F. Gieras, G. E. Dawson and A. R. Eastham, "A new longitudinal end effect factor for linear induction motors," IEEE Transaction on Magnetics, EC-2(1), pp.152-159,1987.

[5] Sung Chan Ahn, Jung Ho Lee, Dong Seok Hyun, "Dynamic characteristic analysis of LIM using coupled FEM and control algorithm," IEEE Trans. Magnetics, Vol.36, No.4, July 2000.

[6] J. F. Gieras, Linear induction drives, Oxford: Clarendon, 1994.

[7] S.A. Nasar and I.Boldea, Electric drives, CRC Press, 1999, pp.210-225.

[8] I. Boldea and S. A. Nasar, Linear motion electromagnetic systems, New York: Wiley,1985, pp.160-250..

[9] J.-H. Sung and K. Nam, "A new approach to vector control for a linear induction motor considering end effects," IEEE Trans on Magnetics, Vol.213, pp.2284-2289,1999.

[10] K. Venkataratnam and A. B. Chattopadhyay, "Analysis of electromagnetic forces in a levitated short rotor LIM-Part II: lateral stabilization," IEEE Trans. on Energy Conversion, Vol.17, No.1, pp. 102-106, March 2002.

[11] S. Yamamura, Theory of linear induction motors, Tokyo Press, 1978.

[12] S. Yamamura, H. Ito and Y. Ishulawa, "Theories of the Linear induction motor and compensated linea induction motor", IEEE Trans on Power App. and Systems, Vol. PAS-91, No. 4, pp 1700-1710, 1971.

[13] X. Long, Theory and magnetic design method of linear induction motor (in Chinese), China: Science Publishing Company, 2006.

\section{APPENDIX}

\section{List of principal symbols}

$\sigma \quad$ Conductivity of secondary sheet

$\omega \quad$ Angular frequency of power supply

$\tau_{\mathrm{e}} \quad$ Half-wave length of end-effect wave

$\alpha_{1} \quad$ Length of entry-end-effect wave penetration coefficient

$\alpha_{2} \quad$ Length of exit-end-effect wave penetration coefficient

$\varepsilon_{0} \quad$ Primary phase inducted electromotive force

$\tau \quad$ Pole pitch in $\mathrm{m}$ (or half wave length)

$\tau_{e} \quad$ End-effect half wave length

$b_{\text {y }} \quad y$-axis magnetic flux density

$g \quad$ Equivalent air-gap length

$g_{\mathrm{m}} \quad$ Mechanical air gap length

$l_{\mathrm{w}} \quad$ Width of primary lamination

Phase number

Per-unit slip

$v_{\mathrm{s}} \quad$ Motor synchronous speed

$w_{1} \quad$ Number of turns per phase for primary winding

$B_{0} \quad$ Amplitude for magnetic flux density of normal wave

$B_{1} \quad$ Amplitude for magnetic flux density of entry-end-effect wave

$B_{2} \quad$ Amplitude for magnetic flux density of exit-end-effect wave

$E_{0} \quad$ Primary induced electromotive force per phase by normal electric field intensity

$E_{1} \quad$ Primary induced electromotive force per phase by entry-end electric field intensity

$E_{2} \quad$ Primary induced electromotive force per phase by exit-end electric field intensity

$F_{\mathrm{e} 0} \quad$ Fundamental-wave thrust

$F_{Q} \quad$ Fundamental-wave mechanical thrust

$F_{\text {el }} \quad$ Entrance-wave thrust

$F_{\mathrm{e} 2} \quad$ Exit-wave thrust

$G \quad$ Goodness factor

$J_{1} \quad$ Equivalent primary current sheet

$L_{\mathrm{p}} \quad$ Primary length

$K_{\mathrm{t}} \quad$ Transverse end correct coefficient

$K_{\mathrm{b}} \quad$ Air gap flux density correct coefficient

$K_{\mathrm{p}} \quad$ Half-filled slot correct coefficient

$K_{\mathrm{w} 1} \quad$ Winding coefficient of primary winding

$P_{\mathrm{m} 1} \quad$ Electromagnetic power by entrance flux density wave

$P_{\mathrm{m} 2} \quad$ Electromagnetic power by exit-end flux density wave

$X_{\mathrm{m}} \quad$ Phase magnetizing reactance

$Z_{\mathrm{m}} \quad$ Primary Phase magnetizing impedance

$\boldsymbol{Z}_{\mathrm{L}} \quad$ Total equivalent longitudinal end-effect impedance

$\boldsymbol{Z}_{\mathrm{Lc}} \quad$ Correct longitudinal end-effect impedance

$\boldsymbol{Z}_{\mathrm{mc}}$ Correct fundamental impedance

$\boldsymbol{Z}_{\mathrm{t}} \quad$ Total circuit impedance 